\title{
Loop induced singlet scalar production through the vector like top quark at future lepton colliders
}

\author{
Daruosh Haji Raissi ${ }^{1}$, Seddigheh Tizchang ${ }^{2}$, and Mojtaba Mohammadi Najafabadi ${ }^{2}$ \\ ${ }^{1}$ Faculty of Sciences, Department of Physics, Ayatollah Amoli Branch, Islamic Azad University, Amol, \\ Mazandaran, Iran \\ ${ }^{2}$ School of Particles and Accelerators, Institute for Research in Fundamental Sciences (IPM) P.O. Box \\ 19395-5531, Tehran, Iran
}

\begin{abstract}
In this paper, we explore the signature of a simplified model which includes a new singlet scalar state and a vector like quark at future lepton colliders. In particular, we study the production of the new singlet scalar in association with a photon, which proceeds through loop level diagrams involving vector like top quark partner, at future $e^{-} e^{+}$colliders with different center-of-mass energies from $500 \mathrm{GeV}$ to $3 \mathrm{TeV}$. To show the sensitivity of the process, the exclusion limits on the parameter space of the model are presented considering the decay of the singlet scalar into a pair of Higgs boson, followed by the decay of Higgs bosons into $b \bar{b}$ pairs. The results are compared to those obtained from the LHC, electroweak precision data and other channels at lepton colliders and it is shown that a notable sensitivity to the parameter space of the simplified model could be achieved.
\end{abstract}




\section{Introduction}

With the Higgs boson discovery at Run I of the LHC [1,2], followed by measurements of its properties from the LHC second run $[36]$, the spontaneous symmetry breaking mechanism of the Standard Model (SM) is confirmed. However, there are still unanswered questions in the SM framework which motivates proposing theoretical models beyond the SM. Among them, there are models with extended scalar and fermion sectors of the SM, which are well motivated to control the instability of electroweak vacuum $[7,9]$ and hierarchy problem [10,11. In particular, models of extended quark sector with vector like quarks (VLQs) appear in composite Higgs models 12, 13, extra dimensions 14, little Higgs models [15], gauging of the flavor group 16, and non-minimal supersymmetric SM [17]. In this work, the concentration is on a simplified model with minimal fields content and interactions, which adds a singlet scalar and a vector like top quark to the SM. In the considered scenario, both the new scalar and vector like top quark mix with the Higgs boson and top quark, respectively [9, 18].

In the SM context, based on the next-to-next-to-leading calculation, the Higgs boson self coupling $\lambda$ tends to be negative at a high energy scale of around $10^{10} \mathrm{GeV}$ which causes instability of the Higgs vacuum. The vacuum stability is quite dependent on how precise the top quark mass is measured. It is notable that the current measured values of the Higgs boson and top quark masses suggest that the vacuum could be metastable [19 23]. In the considered simplified model, the presence of the new scalar can have a positive contribution to the Higgs boson quartic coupling $\lambda$ and consequently could push the Higgs boson potential toward the stable phase. It is worth mentioning that the added VLQ in this minimal model will help preserve the perturbativity of the new scalar quartic coupling [9].

There are tight bounds on the couplings of the light quarks to the heavy vector like quarks from flavor physics [24], as a result in this simplified model no interaction among the vector like quark and the first two quark generations is considered. The new scalar state couples to the massive SM particles through its mixing with Higgs boson and couples to the massless photon and gluon via loops involving the VLQ and top quark $[9,18,25,32$.

So far, no evidence for existing such new particles have been observed by the collider experiments. There are studies for vector like top quark $T$ at the LHC by the ATLAS and CMS experiments, where the searches have been performed for vector like top quark pair $T \bar{T}$ production through strong interactions 3335$]$. In these searches, the $T$ and $\bar{T}$ quarks are assumed to decay into $t Z, t H$, and $W b$ and the searches have been performed in different channels like single lepton, multilepton, and full hadronic. In all studied final states, the results are compatible with the SM expectations hence limits have been set on the model parameters. Other studies on constraining the masses, couplings of the vector like top quark $T$, and the new scalar can be found in Refs. 18, 36 42. Model-independent approach using effective operators for the vector like top quark partner has been studied in Ref. [43]. The vector like top quark $T$ can also be produced singly in association with a light quark and either a top quark or a bottom quark at the LHC which has been the subject of several studies such as Refs. 44, 45].

In addition to the direct searches, the parameter space of the simplified model with a singlet scalar and VLQ has been probed using the electroweak precision observables as well as the Higgs coupling precision measurements that could be found in Ref. [9]. The requirement of vacuum stability and the unitarity of the VLQ and scalar scatterings constrain the parameter space. Among all the limits, those from vacuum stability are the tightest 9 .

The future electron-positron colliders such as International Linear Collider (ILC) [46, 47], Compact Linear Collider (CLIC) with a possibility of going to high center-of-mass energy up to $3 \mathrm{TeV}$ [48, the Future Circular Collider FCC-ee [4, 50, and Circular Electron-Positron Collider 
CEPC [51] provide very clean places to measure the SM parameters and to search for new physics effects. Lack of hadronic initial state, low amount of background, and accurate knowledge of initial beam energy would flatten the way of precision studies or discovery of new particles.

The goal of the present work is to study the phenomenology of the direct production of the singlet scalar at the future lepton colliders and inquire its effects on the phenomenology of lepton colliders. In particular, the production of the singlet scalar in association with a photon is studied because the final state has an energetic photon and could be used as a handle to reduce the background contributions and trigger the signal events. The process proceeds through loop level diagrams where the VLQ contributes to the loops, therefore the channel is sensitive to the related parameters of the VLQ and would be a complementary process to the other channels to search for the model. This paper is organized as the following: In section 2 a brief review of the model and its main properties are given. Section 3 provides a phenomenological study on the new scalar associated with a photon at the high energy lepton colliders. In section 4 , the possible final states and potential bounds on the model parameters are presented. The results are compared with the constraints obtained from the LHC data and from the expectation of the single scalar production channel at a future high energy lepton collider. Section 5 is devoted to the summary and conclusions.

\section{The model}

In this section, a brief description of the simplified model where the SM is extended by adding a new neutral singlet scalar $S$ and a vector like quark $T$ is given. The VLQ $T$ carries the same quantum numbers as the right-handed top quark and mixes only with the SM top quark. More detailed description of the model could be found in Refs. [9, 18. In the new scenario, the scalar, Yukawa and gauge sectors of the SM Lagrangian receive changes:

$$
\mathcal{L} \supset \mathcal{L}_{\text {scalar }}+\mathcal{L}_{\text {Yukawa }}+\mathcal{L}_{\text {gauge }}
$$

where

$$
\begin{aligned}
\mathcal{L}_{\text {scalar }} & =\frac{1}{2}\left(D_{\mu} H\right)^{\dagger}\left(D^{\mu} H\right)+\frac{1}{2} \partial_{\mu} S \partial^{\mu} S-\mu^{2} H^{\dagger} H+\lambda\left(H^{\dagger} H\right)^{2} \\
& +\frac{a_{1}}{2} H^{\dagger} H S+\frac{a_{2}}{2} H^{\dagger} H S^{2}+b_{1} S+\frac{b_{2}}{2} S^{2}+\frac{b_{3}}{3} S^{3}+\frac{b_{4}}{4} S^{4},
\end{aligned}
$$

here $H$ is the SM Higgs boson doublet and the new scalar field is denoted by $S$. To keep the Yukawa term $S \bar{T} T$, no $Z_{2}$ symmetry is applied however conventionally $a_{1}, b_{1}, b_{3}$ are set to zero. With such assumptions on the couplings, one can explain all the relevant measurements and also explain the motivations for which the model has been proposed. Both the SM Higgs doublet and new scalar $S$ acquire non-zero vacuum expectation values:

$$
H=\left(\begin{array}{c}
i \phi^{+} \\
\frac{1}{\sqrt{2}}\left(v_{H}+h+i \phi^{0}\right)
\end{array}\right), \quad S=\left(s+v_{S}\right),
$$

where $v_{H}$ is the vacuum expectation value (vev) of the SM Higgs boson and is $\approx 246 \mathrm{GeV}$ and

the vev of the singlet scalar is denoted by $v_{S}$. After the spontaneous symmetry breaking and expanding the Lagrangian around its minimum, the squared mass matrix has the following form:

$$
\mathcal{M}_{\text {scalar }}^{2}=\left(\begin{array}{cc}
2 \lambda v_{H}^{2} & a_{2} v_{H} v_{S} \\
a_{2} v_{H} v_{S} & 2 b_{4} v_{S}^{2}
\end{array}\right)
$$


and the squared masses of the physical eigenstates are found to be:

$$
m_{h_{1}, h_{2}}^{2}=\lambda v_{H}^{2}+b_{4} v_{S}^{2} \mp \sqrt{\left(b_{4} v_{S}^{2}-\lambda v_{H}^{2}\right)^{2}+a_{2}^{2} v_{H}^{2} v_{S}^{2}} .
$$

The physical eigenstates $h_{1,2}$ are related to the singlet scalar field $s$ and the SM Higgs field $h$ through the following transformation:

$$
\left(\begin{array}{l}
h_{1} \\
h_{2}
\end{array}\right)=\left(\begin{array}{cc}
\cos (\theta) & -\sin (\theta) \\
\sin (\theta) & \cos (\theta)
\end{array}\right)\left(\begin{array}{l}
h \\
s
\end{array}\right)
$$

where $\theta$ is the mixing angle and is defined as:

$$
\tan (2 \theta)=\frac{a_{2} v_{H} v_{S}}{b_{4} v_{S}^{2}-\lambda v_{H}^{2}} .
$$

It is assumed that the $h_{1}$ scalar field is the SM Higgs boson with a mass of $m_{h_{1}}=125 \mathrm{GeV}$, which will denoted as $h$ afterhere. To have the vacuum stability at large energy scales, the mixing angle $\theta$ has to be small [52,53]. Considering the LHC measurements of the Higgs boson, electroweak precision data, and respecting the vacuum stability conditions impose that $|\sin (\theta)| \lesssim 0.2$.

The third quark generation Yukawa part of the Lagrangian is modified as follows:

$$
\mathcal{L}_{\text {Yukawa }}=y_{T} S \bar{T}_{L}^{\mathrm{int}} T_{R}^{\mathrm{int}}+y_{t} \bar{Q}_{L}^{\mathrm{int}} \widetilde{H} t_{R}^{\mathrm{int}}+y_{b} \bar{Q}_{L}^{\mathrm{int}} H b_{R}+\lambda_{T} \bar{Q}_{L}^{\mathrm{int}} \widetilde{H} T_{R}^{\mathrm{int}},
$$

where int index stands for weak interaction eigenstates, $\bar{Q}_{L}$ is left-handed third generation quark doublet, $\bar{Q}_{L}=\left(\vec{t}_{L}^{\text {int }} \bar{b}_{L}^{\text {int }}\right)$ and $\widetilde{H}=i \sigma_{2} H^{*}$. We note that one could add a Dirac mass term for vector like top quark $T$ to the above Lagrangian however after the spontaneous symmetry breaking $T$ acquires mass. Therefore, in order to reduce the number of free parameters in the model, Dirac mass term is not added to the Lagrangian. A term proportional to $\bar{T}_{L}^{\text {int }} t_{R}^{\text {int }}$, can also be present in the $\mathcal{L}_{\text {Yukawa }}$, however, it can be removed by a redefinition of the right-handed fields $\left(t_{R}^{\text {int }}, T_{R}^{\text {int }}\right)[54]$. After the spontaneous symmetry breaking, the SM top quark $t^{\text {int }}$ and vector like top quark $T^{\text {int }}$ mix and the mass matrix can be written as:

$$
\mathcal{M}_{\text {Yukawa }}=\left(\begin{array}{cc}
y_{t} v_{H} / \sqrt{2} & \lambda_{T} v_{H} / \sqrt{2} \\
0 & y_{T} v_{S}
\end{array}\right) .
$$

The mass matrix is diagonalized by the unitary transformation with $\theta_{L}$ and $\theta_{R}$ rotation angles:

$$
\left(\begin{array}{c}
t_{L / R} \\
T_{L / R}
\end{array}\right)=\mathbf{U}_{L / R}\left(\begin{array}{c}
t_{L / R}^{\mathrm{int}} \\
T_{L / R}^{\mathrm{int}}
\end{array}\right),
$$

where $t_{L / R}$ and $T_{L / R}$ are the physical mass eigenstates and the unitary matrices are written as:

$$
\mathbf{U}_{L / R}=\left(\begin{array}{cc}
\cos \theta_{L / R} & -\sin \theta_{L / R} \\
\sin \theta_{L / R} & \cos \theta_{L / R}
\end{array}\right)
$$

The squared mass eigenvalues for the SM top quark and the vector like quark $T$ are found to be:

$$
m_{T, t}^{2}=\frac{1}{4}\left(y_{t}^{2} v_{H}^{2}+\lambda_{T}^{2} v_{H}^{2}+2 y_{T}^{2} v_{S}^{2} \pm \sqrt{\left(y_{t}^{2} v_{H}^{2}+\lambda_{T}^{2} v_{H}^{2}+2 y_{T}^{2} v_{S}^{2}\right)^{2}-8 y_{t}^{2} v_{H}^{2} y_{T}^{2} v_{S}^{2}}\right)
$$

where the lighter eigenstate is assumed to be the SM top quark with $m_{t}=173.2 \mathrm{GeV}$ and the heavier is taken as the vector like $T$ with a mass $m_{T}$. Two mixing angles are related through the following relation [55]:

$$
\tan \left(\theta_{R}\right)=\frac{m_{t}}{m_{T}} \tan \left(\theta_{L}\right)
$$


It is clear that the left-handed mixing angle is always dominant, in particular for the heavy vector like $T$. The Yukawa coupling terms, mass terms of the $t, T$ and the mixing term between $t$ and $T$ are given by:

$$
\begin{aligned}
\mathcal{L}_{\text {Yukawa }} & \supset \frac{m_{T}}{v_{H} v_{S}}\left(s_{L}^{2} v_{S}\left(h-i \phi^{0}\right)+c_{L}^{2} v_{H} s\right) \bar{T}_{L} T_{R} \\
& +\frac{m_{t}}{v_{H} v_{S}}\left(c_{L}^{2} v_{S}\left(h-i \phi^{0}\right)+s_{L}^{2} v_{H} s\right) \bar{t}_{L} t_{R} \\
& +\frac{m_{T}}{v_{H} v_{S}} s_{L} c_{L}\left(v_{S}\left(h-i \phi^{0}\right)-v_{H} s\right) \bar{t}_{L} T_{R} \\
& +\frac{m_{t}}{v_{H} v_{S}} s_{L} c_{L}\left(v_{S}\left(h-i \phi^{0}\right)-v_{H} s\right) \bar{T}_{L} t_{R},
\end{aligned}
$$

where $s_{L}\left(c_{L}\right) \equiv \sin \theta_{L}\left(\cos \theta_{L}\right)$. Electroweak gauge interactions of vector like quark $T$ with the quantum number $Q_{T}=2 / 3$ and $Y_{T}=4 / 3$ with the SM third quark generation $t, b$ are as follows:

$$
\begin{aligned}
\mathcal{L}_{\text {gauge }} & \supset i \bar{t} \not \partial t+i \bar{b} \not \partial b+i \bar{T} \not \partial T \\
& +e\left(Q_{t} \bar{t} \gamma^{\mu} t+Q_{T} \bar{T} \gamma^{\mu} T+Q_{b} \bar{b} \gamma^{\mu} b\right) A_{\mu} \\
& +\frac{g}{\sqrt{2}}\left(\left(c_{L} \bar{t} \gamma^{\mu} P_{L} b+s_{L} \bar{T} \gamma^{\mu} P_{L} b\right) W_{\mu}^{+}+\left(c_{L} \bar{b} \gamma^{\mu} P_{L} t+s_{L} \bar{b} \gamma^{\mu} P_{L} T\right) W_{\mu}^{-}\right) \\
& +\frac{g}{c_{w}}\left(\bar{T} \gamma_{\mu}\left(\frac{s_{L}^{2}}{2} P_{L}-Q_{T} s_{w}^{2}\right) T+\bar{t} \gamma_{\mu}\left(\frac{c_{L}^{2}}{2} P_{L}-Q_{t} s_{w}^{2}\right) t\right. \\
& \left.+\bar{b} \gamma_{\mu}\left(-\frac{1}{2} P_{L}-Q_{b} s_{w}^{2}\right) b+\bar{t} \gamma_{\mu} \frac{s_{L} c_{L}}{2} P_{L} T+\bar{T} \gamma_{\mu} \frac{s_{L} c_{L}}{2} P_{L} t\right) Z_{\mu},
\end{aligned}
$$

where $\theta_{w}$ is Weinberg weak mixing angle, $s_{w}\left(c_{w}\right) \equiv \sin \theta_{w}\left(\cos \theta_{w}\right)$ and $P_{L}=\left(1-\gamma_{5}\right) / 2$ is projection operator. More explanation for driving of the above interactions are given in appendix of Ref. [18]. The simplified model followed here has five unknown parameters which consists of the mass of vector like top quark $m_{T}$, the new singlet scalar mass $m_{h_{2}}$, vacuum expectation value of the singlet scalar $v_{S}$, and the mixing angles $\theta_{L}$ and $\theta$ in the fermion and scalar sectors, respectively.

In Ref. [56], the production of a new scalar singlet $h_{2}$ has been studied via vector boson fusion $\left(e^{-}+e^{+} \rightarrow h_{2}+\nu \bar{\nu}\right)$ at a high energy lepton collider with the center-of-mass energy of $3 \mathrm{TeV}$. In particular, it has been found that a future high energy lepton collider would be able to examine the single scalar production rate with a few tens of atto-barn. The production of the scalar $h_{2}$ associated with a $Z$ boson at a lepton collider operating at the center-of-mass energy of 240 $\mathrm{GeV}$ has been studied in Ref. [57]. In section 3, we calculate the production cross section of the scalar $h_{2}$ in association with a photon at the lepton colliders. The cross section is presented at different center-of-mass energies of the electron-positron collisions and its dependence on the free parameters of the model is presented.

\section{Singlet scalar production in association with a photon}

In this section, we propose an alternative way to have access to parameter space of the simplified model by considering the process $e^{-} e^{+} \rightarrow h_{2} \gamma$ which proceeds via loops with contributions from SM fermions, gauge bosons and, the VLQ. In Ref. [58], the cross section of the associated production of a photon and a Higgs boson in the context of Minimal Supersymmetric Standard Model (MSSM) has been calculated. The production rates for the associated production of both the CP-even and CP-odd Higgs bosons of the MSSM have been studied. For the CP-even MSSM Higgs boson production with a photon, other s-channel Feynman diagrams involving loops with 

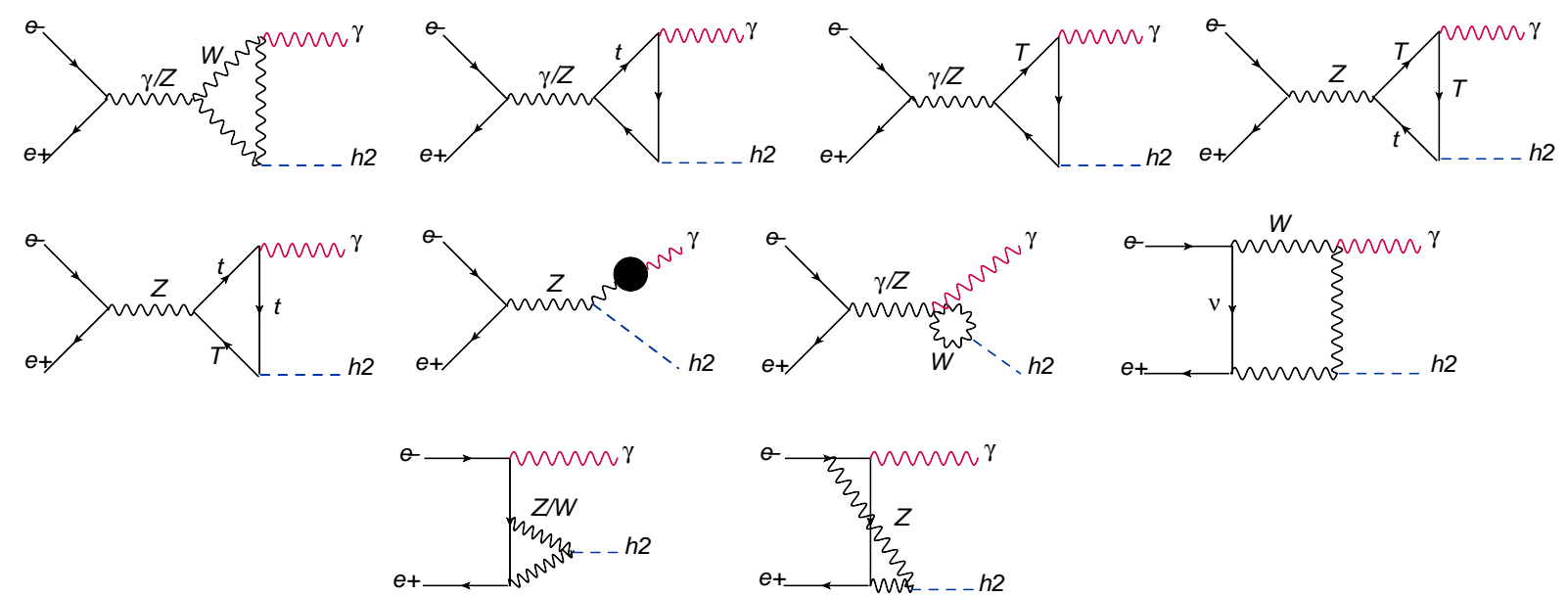

Figure 1: Representative Feynman diagrams for the process $e^{-} e^{+} \rightarrow h_{2} \gamma$ in the context of simplified model.

charginos, charged Higgs bosons, squarks, and sleptons appear and in the t-channel box diagrams chargino/sneutrino and neutralino/selectron contribute to the production process. The production rate of the Higgs boson in association with a photon at electron-positron colliders, in the context of extended Higgs models, like the two-Higgs-doublet model, the inert doublet model, and the inert triplet model (ITM) has been studied in Ref. [59]. The authors found that the charged scalars of these models via loop diagrams can generate sizable contributions to the production cross section of $h+\gamma$. The potential of the LHC to probe the new physics effects in the SMEFT (SM Effective Field Theory) framework through the Higgs boson production associated with a photon has been studied in Ref. [60]. The next-to-leading order QCD corrections to the production of a Higgs boson associated with a photon has been calculated in Ref. 61, where it has been shown that these corrections could increase the production rate up to $20 \%$.

Within the simplified model, the $e^{-} e^{+} \rightarrow h_{2} \gamma$ process proceeds through loop-level Feynman diagrams which include SM fermions and gauge bosons as well as the additional contributions from the vector like top quark. Representative Feynman diagrams contributing to the process $e^{-} e^{+} \rightarrow h_{2} \gamma$ are presented in Fig,1. There are s-channel diagrams with $Z / \gamma$ boson exchange in which virtual $W$ boson, heavy SM fermion (mostly top and bottom quarks) as well as the vector like top quark in the loops are involved. The new singlet scalar $h_{2}$ couples to the top quark via both the fermion and scalar mixing which causes triangle diagrams in the s-channel production. The t-channel Feynman diagrams involve $W, Z, \nu_{e}$ and electron exchanges. Contributions from s-channel diagrams with the SM Higgs boson $h$ and its interference with the $Z$ boson or photon is negligible.

Since the process occurs at higher order electroweak interaction, the cross section is expected to be rather small, however the signal is very clean specially due to the presence of an energetic photon in the final state. This would allow to achieve a reasonable background suppression and leads to have a good sensitivity in particular using the expected large amount of data by the future lepton colliders.

Within the considered simplified model in this work, the one-loop amplitude, neglecting the mass of electron, for the $h_{2}+\gamma$ production can be written as the sum of the amplitudes of all contributing diagrams:

$$
\mathcal{M}=\sum_{k=1,2} \sum_{v=+,-} \Lambda_{k}^{v} C_{k}^{v}
$$


where $\Lambda_{k}^{ \pm}$have the following form 58 :

$$
\begin{aligned}
& \Lambda_{1}^{ \pm}=\bar{v}\left(q_{+}\right)\left(1 \pm \gamma_{5}\right)\left(\xi_{\gamma}^{*} p_{\gamma} \cdot q_{-}-\not p_{\gamma} \epsilon_{\gamma}^{*} \cdot q_{-}\right) u\left(q_{-}\right) \\
& \Lambda_{2}^{ \pm}=\bar{v}\left(q_{+}\right)\left(1 \pm \gamma_{5}\right)\left(\xi_{\gamma}^{*} p_{\gamma} \cdot q_{+}-p_{\gamma} \epsilon_{\gamma}^{*} \cdot q_{+}\right) u\left(q_{-}\right),
\end{aligned}
$$

where $q_{ \pm}$are the momenta of the $e^{ \pm}$beams, $p_{\gamma}$ is the four-momentum of external photon and $\epsilon_{\gamma}$ is the corresponding polarization vector and $C_{k}^{ \pm}$represent form factor coefficients obtained by summing the diagrams depicted in Fig,1:

$$
C_{k}^{ \pm}=C_{k}^{\gamma^{ \pm}}+C_{k}^{Z \pm}+C_{k}^{ \pm \text {Wbox }}+C_{k}^{ \pm \text {Zbox }}
$$

where $C_{k}^{\gamma, \pm}$ and $C_{k}^{Z, \pm}$ represent the contributions of $\gamma$ and $Z$ propagators in s-channel diagrams for vertex corrections and $C_{k}^{ \pm \text {Wbox }}$ and $C_{k}^{ \pm \text {Zbox }}$ are the contributions of box diagrams. The total cross section for unpolarized beam is obtained as:

$$
\frac{\mathrm{d} \sigma}{\mathrm{d} \cos \theta_{s}}=\frac{s-m_{h_{2}}^{2}}{64 \pi s} \frac{1}{\left(16 \pi^{2}\right)^{2}}\left[u^{2}\left(\left|C_{1}^{+}\right|^{2}+\left|C_{1}^{-}\right|^{2}\right)+t^{2}\left(\left|C_{2}^{+}\right|^{2}+\left|C_{2}^{-}\right|^{2}\right)\right]
$$

where $\theta_{s}$ is the the angle between the incoming electron and outgoing photon. To obtain the above differential cross section, an averaging over the helicities of the incoming leptons and a sum over the outgoing photon polarization has been performed. $s, t$ and $u$ are the Mandelstam variables and are defined as $s=\left(q_{-}+q_{+}\right)^{2}, t=\left(q_{+}-p_{\gamma}\right)^{2}$ and $u=\left(q_{-}-p_{\gamma}\right)^{2}$. The $C_{k}^{ \pm}$coefficients can be written as a function of Passarino-Veltman functions. The $C_{k}^{\gamma \pm}$ coefficient contains contribution from three diagrams involving $W$ boson, top quark and vector like top quark in the loop-induced vertex $h_{2} \gamma \gamma$ and the $C_{k}^{Z \pm}$ includes $W$ boson, top quark and vector like top quark contribution in loop-induced vertex $h_{2} Z \gamma . C_{k}^{\gamma \pm}$ and $C_{k}^{Z \pm}$ have the following forms:

$$
\begin{aligned}
& C_{1}^{\gamma \pm}=C_{2}^{\gamma \pm}=-\frac{e}{2} \frac{1}{s} G^{\gamma}, \\
& C_{1}^{Z \pm}=C_{2}^{Z \pm}=-\frac{e z^{ \pm}}{4 s_{w} c_{w}} \frac{1}{s-M_{Z}^{2}} G^{Z},
\end{aligned}
$$

where $e$ is the size of electron electric charge, $z^{+}=-1+2 s_{w}^{2}$, and $z^{-}=2 s_{w}^{2}$. Considering both the bosonic and fermionic contributions in s-channel vertex correction, $G^{\gamma}$ and $G^{Z}$ are obtained 
as follows:

$$
\begin{aligned}
G^{\gamma} & =\frac{e^{3} M_{W}}{s_{w}}\left[F^{\gamma, W} s_{\theta}-\sum_{f} 4 Q_{f}^{2} N_{c} \frac{m_{f}^{2}}{M_{W}^{2}} F^{f} s_{\theta}\right. \\
& \left.-4 F^{t} \frac{m_{t}^{2}}{M_{W}^{2}} N_{c} Q_{t}^{2}\left(r s_{L}^{2} c_{\theta}+c_{L}^{2} s_{\theta}\right)-4 F^{T} \frac{m_{T}^{2}}{M_{W}^{2}} N_{c} Q_{T}^{2}\left(r c_{L}^{2} c_{\theta}+s_{L}^{2} s_{\theta}\right)\right] \\
G^{Z} & =\frac{e^{3} M_{W}}{c_{w} s_{w}^{2}}\left[F^{Z, W} s_{\theta}+\sum_{f} 2 Q_{f} N_{c} \frac{m_{f}^{2}}{M_{W}^{2}}\left(I_{3}^{f}-2 s_{w}^{2} Q_{f}\right) F^{f} s_{\theta}\right. \\
& +2 Q_{t} N_{c} \frac{m_{t}^{2}}{M_{W}^{2}}\left(I_{3}^{t} c_{L}^{2}-2 s_{w}^{2} Q_{t}\right)\left(c_{L}^{2} s_{\theta}+r s_{L}^{2} c_{\theta}\right) F^{t} \\
& +2 Q_{T} N_{c} \frac{m_{T}^{2}}{M_{W}^{2}}\left(I_{3}^{T} s_{L}^{2}-2 s_{w}^{2} Q_{T}\right)\left(s_{L}^{2} s_{\theta}+r c_{L}^{2} c_{\theta}\right) F^{T} \\
& +2 Q_{t} N_{c} \frac{m_{t}+m_{T}}{M_{W}^{2}} s_{L}^{2} c_{L}^{2}\left(s_{\theta}-r c_{\theta}\right) F^{n} \\
& \left.+2 Q_{T} N_{c} \frac{m_{t}+m_{T}}{M_{W}^{2}} s_{L}^{2} c_{L}^{2}\left(s_{\theta}-r c_{\theta}\right) F^{l}\right]
\end{aligned}
$$

where $m_{f}, Q_{f}$ and $I_{3}^{f}$ are the mass, electric charge and third component of weak isospin of the fermion $f$ ( $f$ can be all fermions except for $t$ and $T$ ), respectively. $N_{c}$ is the number of QCD colors and $r \equiv v_{H} / v_{S}$ and $s_{\theta}\left(c_{\theta}\right) \equiv \sin \theta(\cos \theta)$. Functions $F$ with various indices in Eq 20 are the combination of Passarino-Veltman scalar functions and are given in the Appendix A. In order to ensure all the contributing Feynman diagrams from SM and new physics are consistently included, the gauge invariance of the matrix elements is checked through validating the Ward-Takahashi identity. In the model, the new scalar couples to the SM particles through mixing with the Higgs boson as $h=c_{\theta} h_{1}+s_{\theta} h_{2}$. Therefore, the new scalar coupling to weak gauge bosons and fermions (except the top quark) are similar to the SM Higgs coupling and just receive a correction factor $s_{\theta}$. The scalar coupling to top quark is modified by both mixing scalar and Yukawa top sectors (see Eq, 13). Among the diagrams, the amplitude of box diagrams $\left(C_{k}^{ \pm W, Z \text { box }}\right)$ have the same form as the SM Higgs associated production with photon by only replacing $m_{H} \rightarrow m_{h_{2}}$ and adding $s_{\theta}$ which comes from $V V h_{2}$ coupling. The explicit forms of $C_{k}^{ \pm W, Z \text { box }}$ as a function of PassarinoVeltman scalar functions are given in AppendixA. While in triangle diagrams, vertices $\gamma \gamma h_{2}$ and $Z \gamma h_{2}$ are modified due to the contribution of singlet scalar couplings to top quark, $W$ boson, and the vector like top quark partner. Comparing the box and triangle diagrams contributions, we find the box diagram contributions are expected to be suppressed significantly at small scalar mixing angle. Package-X 62 and LoopTools 63 are used to reduce the tensor integrals and to evaluate the one loop Feynman integrals. The Passarino-Veltman formalism according to Ref. [58] is employed in this work.

The differential cross section $d \sigma\left(e^{-} e^{+} \rightarrow h_{2} \gamma\right) / d \cos \theta_{s}$ for three center-of-mass energies $\sqrt{s}=$ $500 \mathrm{GeV}$ and $1,3 \mathrm{TeV}$ are presented in Fig.2. The distributions are shown for two values of $s_{\theta}= \pm 0.15$ which are denoted by solid $(+0.15)$ and dotted $(-0.15)$ curves. We see that the cross section is not symmetric on $s_{\theta}$ which was expected as the cross section $e^{-} e^{+} \rightarrow h_{2} \gamma$ is proportional to $\propto\left(A s_{\theta}+B c_{\theta}\right)^{2}$. Obviously, the cross section is considerably sensitive to the sign of $s_{\theta}$ and larger cross section is expected for the negative values of $s_{\theta}$ in particular for the low center- of- mass energies of $500 \mathrm{GeV}$ and $1 \mathrm{TeV}$. Moreover, the effect of the sign of $s_{\theta}$ is negligible 
at small scattering angles at $\sqrt{s}=3 \mathrm{TeV}$. One can also see that the angular distribution for both signs of $s_{\theta}$ is symmetric on the scattering angle and no forward-backward asymmetry is expected.

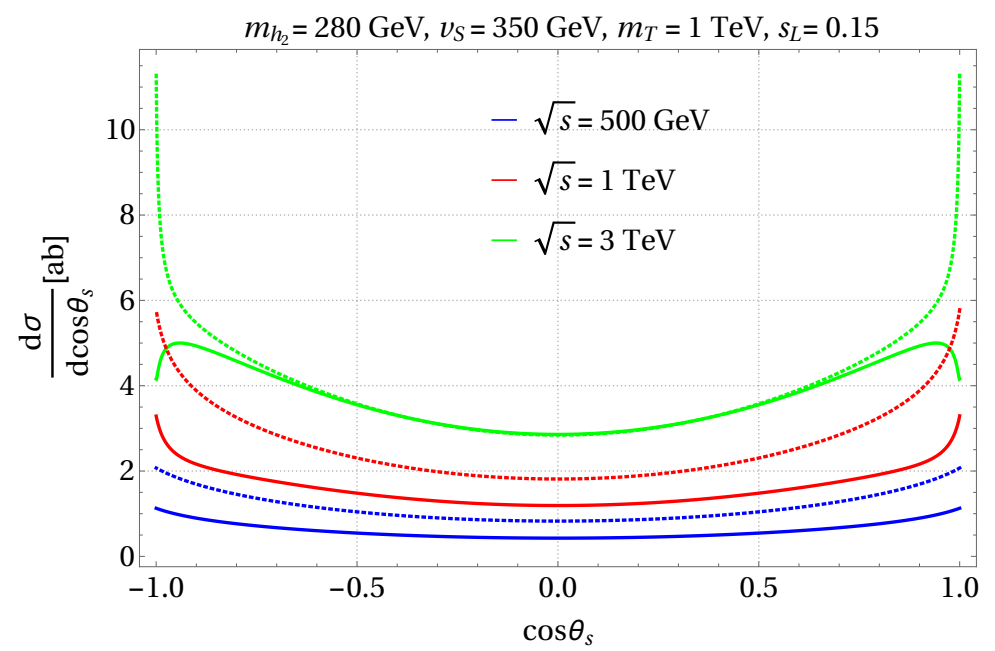

Figure 2: Differential cross section of $h_{2}+\gamma$ production as a function of scattering angle $\cos \theta_{s}$ for $m_{h_{2}}=280 \mathrm{GeV}$ for different center-of-mass energies with $s_{\theta}=0.15$ (solid) and $s_{\theta}=-0.15$ (dotted).

In Fig 3, the total cross section of $e^{-} e^{+} \rightarrow h_{2} \gamma$ is presented as a function of center- of-mass energy $\sqrt{s}$ for $m_{h_{2}}=280 \mathrm{GeV}, s_{L}=0.15$, and $s_{\theta}= \pm 0.15$. The cross sections are shown for two scenarios of assumption on the two sets of free parameters: $\left(m_{T}, v_{S}\right)=(1000,350),(750,400)$ $\mathrm{GeV}$. For both scenarios, the maximum value of cross section occurs at $\sqrt{s} \sim 2 m_{T}$. As can be seen, the cross section increases rapidly up to $T \bar{T}$ threshold then drops slightly with increasing the center-of-mass energy like $1 / s$. It is notable that the contribution of s-channel diagrams to the total cross section is dominant with respect to the box and t-channel diagrams. The impact of the sign of $s_{\theta}$ is explicit in particular for the center-of-mass energies less than $T \bar{T}$ threshold, i.e. $2 m_{T}$. While as the center-of-mass energy increases, the effect of the sign of $s_{\theta}$ is hardly distinguishable. In addition, the cross section enhances when $m_{T}$ goes up.

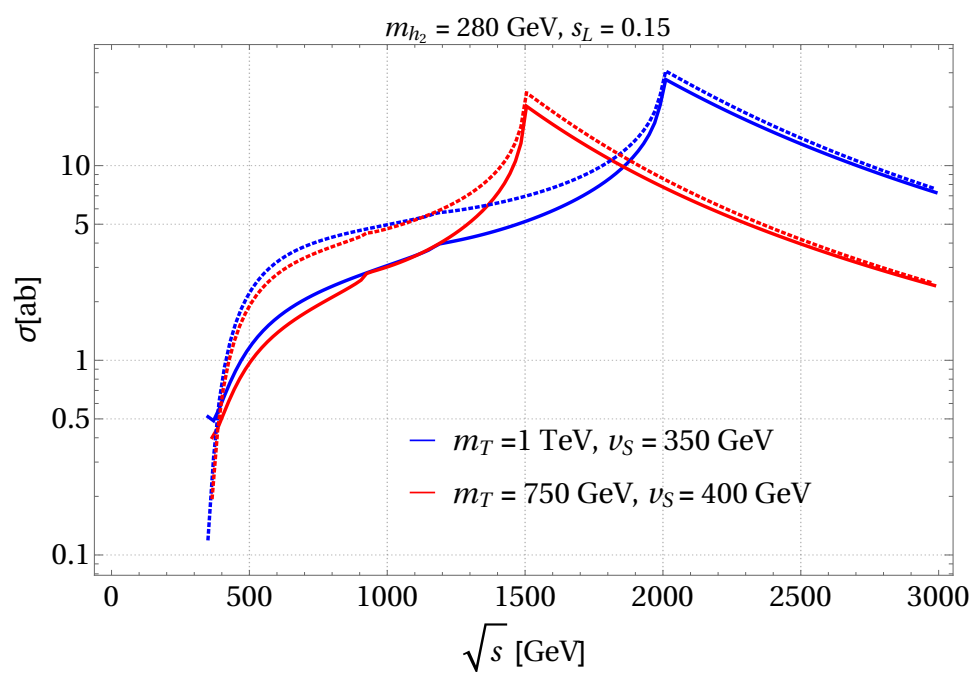

Figure 3: Total cross section of $h_{2}+\gamma$ production as a function of center-of-mass energy $\sqrt{s}$ for different values of $m_{T}$ and $v_{S}$ with $s_{\theta}=0.15$ (solid) and $s_{\theta}=-0.15$ (dotted). 


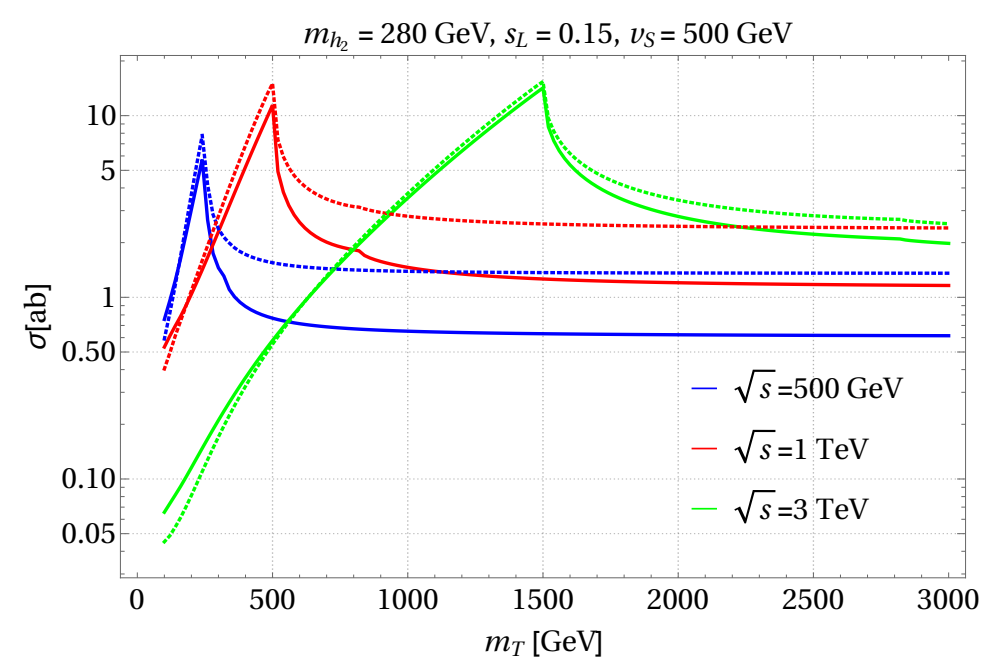

Figure 4: Total cross section of $h_{2}+\gamma$ production as a function of vector like top quark mass, $m_{T}$, for different values of center-of-mass energies with $s_{\theta}=0.15$ (solid) and $s_{\theta}=-0.15$ (dotted).

To illustrate the dependence of the cross section of $e^{-} e^{+} \rightarrow h_{2} \gamma$ process on the mass of vector like top quark, we show the cross section as a function of $m_{T}$ in Fig, 4 . The cross section are shown for three center-of-mass energies of $500 \mathrm{GeV}, 1 \mathrm{TeV}$, and $3 \mathrm{TeV}$ for two cases of the sign of mixing angle of $s_{\theta}= \pm 0.15$. The plot shows that the cross section increases quickly with $m_{T}$ up to $\sim m_{T}=\sqrt{s} / 2$ then it remains almost constant. The cross section is larger for the larger center-of-mass energy. Fig. 5 shows the cross section in terms of of the mass of new scalar $m_{h_{2}}$ for $\sqrt{s}=500 \mathrm{GeV}, 1$ and $3 \mathrm{TeV}$. Because of the larger phase space, the production cross section is large for the low mass of scalar and it decreases by increasing $m_{h_{2}}$. Therefore, more sensitivity is expected to the regions in parameter space with a light scalar. We also note that the cross section decreases more rapidly for the center-of-mass energies of $500 \mathrm{GeV}$ and $1 \mathrm{TeV}$ than the $3 \mathrm{TeV}$ case.
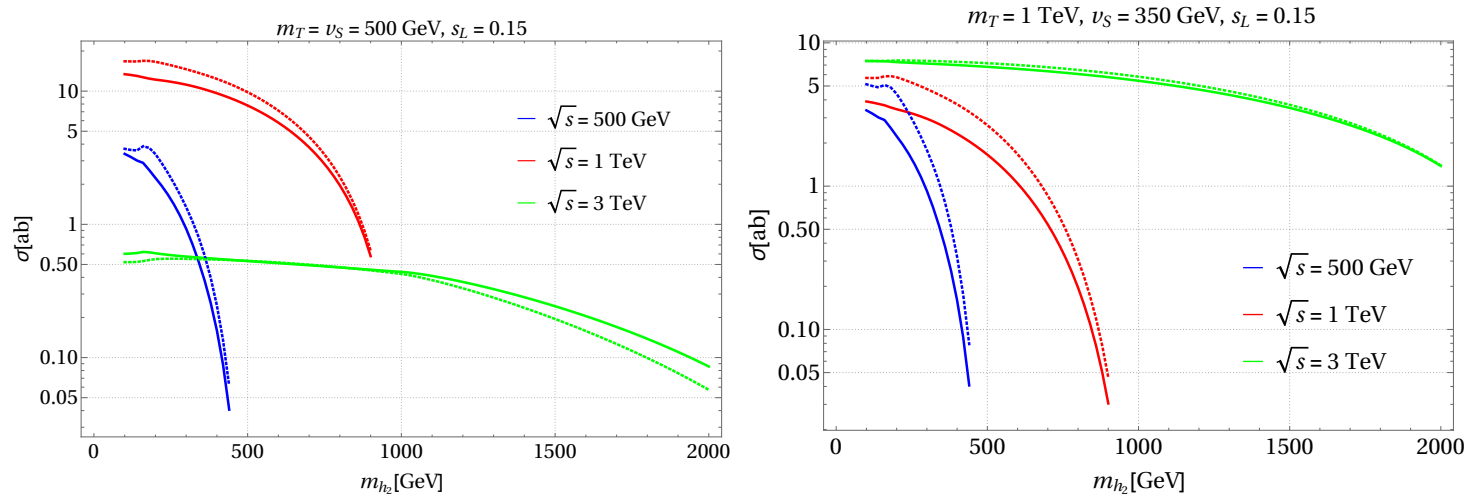

Figure 5: The total cross section of $h_{2}+\gamma$ production as a function of scalar mass $m_{h_{2}}$ for three center-ofmass energies with $m_{T}=v_{S}=500 \mathrm{GeV}$ (left) and $m_{T}=1 \mathrm{TeV}, v_{S}=350 \mathrm{GeV}$ (right) and for $s_{\theta}=0.15$ (solid) and $s_{\theta}=-0.15$ (dotted). 


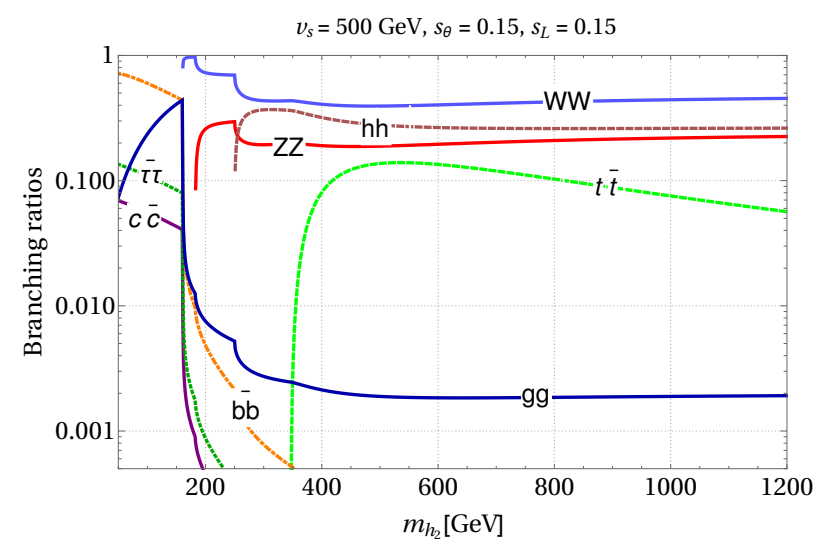

Figure 6: Branching fractions of the new scalar as a function of $m_{h_{2}}$ for $s_{\theta}=s_{L}=0.15$ and $v_{S}=500$ $\mathrm{GeV}$.

Table 1: The $h_{2}$ to $W W, h h, Z Z$, and $t \bar{t}$ decay channels as well as the branching fractions of some of the decay modes.

\begin{tabular}{c|c|c|c}
\hline \hline$Z Z$ mode $[B r \%]$ & $t \bar{t}$ mode $[B r \%]$ & $W W$ mode $[B r \%]$ & $h h$ mode $[B r \%]$ \\
\hline$(l l)\left(l^{\prime} l^{\prime}\right)[1 \%]$ & $\left(l \nu_{l} b\right)\left(q \bar{q}^{\prime} b\right)[44 \%]$ & $\left(l \nu_{l}\right)\left(q \bar{q}^{\prime}\right)[44 \%]$ & $(b \bar{b})(b \bar{b})[34 \%]$ \\
$(l l)(q \bar{q})[14 \%]$ & $\left(l \nu_{l} b\right)\left(l^{\prime} \nu_{l^{\prime}} b\right)[10 \%]$ & $\left(l \nu_{l}\right)\left(l^{\prime} \nu_{l^{\prime}}\right)[10 \%]$ & $(b \bar{b})(l l)[7 \%]$ \\
$(l l)\left(\nu_{l^{\prime}} \nu_{l^{\prime}}\right)[4 \%]$ & $\left(q \bar{q}^{\prime} b\right)\left(q \bar{q}^{\prime} b\right)[46 \%]$ & $\left(q \bar{q}^{\prime}\right)\left(q \bar{q}^{\prime}\right)[46 \%]$ & $(l l)(l l)[0.4 \%]$ \\
$(q \bar{q})\left(q^{\prime} \bar{q}^{\prime}\right)[49 \%]$ & $\ldots \ldots$ & $\ldots \ldots$ & $(b \bar{b})(\gamma \gamma)[0.3 \%]$ \\
$(q \bar{q})(\nu \bar{\nu})[28 \%]$ & $\ldots \ldots$ & $\ldots$. & $(l l)(\gamma \gamma)[0.03 \%]$ \\
$(\nu \bar{\nu})(\nu \bar{\nu})[4 \%]$ & $\ldots \ldots$ & $\ldots .$. & $(W W)(W W)[5 \%]$ \\
\hline \hline
\end{tabular}

\section{Experimental signatures and possible constraints}

There are various direct searches for the new scalar $h_{2}$ at the hadron and lepton colliders. Of particular interest in this work is to perform the search for $h_{2}$ via its production in association with a photon at the future lepton colliders.

Based on the decay modes of $h_{2}$, different topologies are expected to be produced. The $h_{2}$ decay mechanism is almost similar to the Higgs boson, dominated by the $b \bar{b}$ pair and two gluons in low mass region while the decay channels $W W, Z Z, t \bar{t}$, and $h h$ are dominant at large mass region. For more illustration, the branching fractions of the $h_{2}$ decays into the SM particles are shown in Fig 6 for $v_{S}=500 \mathrm{GeV}$ and $s_{\theta}=s_{L}=0.15$. With increasing $m_{h_{2}}$, decay modes of $W W$, $Z Z$, and $h h$ are dominant with respect to $t \bar{t}$. From Fig.6, one can see that at $m_{h_{2}} \geq 200 \mathrm{GeV}$, decay channels $h_{2} \rightarrow g g / \gamma \gamma / f \bar{f}$, where $f$ denotes all fermion except top quark, are negligible.

In this section, we derive potential bounds on the free parameters of the simplified model in $e^{-} e^{+}$colliders through $h_{2}+\gamma$ final state and compare them with the ones obtained from Higgs and precision electroweak data measured at the LHC [18]. Given the interested mass range of $h_{2}$, some of the appropriate, dominant, and clean decay modes are listed in Table 1 as a reference for discussion of the experimental signatures.

In order to make an estimation of the potential of $e^{-} e^{+} \rightarrow h_{2}+\gamma$ process to probe the parameter space of the model, we consider the $h_{2}$ decay into $h h$, followed by $h \rightarrow b \bar{b}$, i.e. $e^{-} e^{+} \rightarrow$ $h_{2} \gamma \rightarrow h h \gamma \rightarrow b \bar{b} b \bar{b} \gamma$. These choices are made due to the large branching fractions of $h_{2} \rightarrow h h$ and $h \rightarrow b \bar{b}$. The cross section of signal including the whole decay chain is computed as $\sigma\left(e^{+} e^{-} \rightarrow\right.$ 
$\left.h_{2} \gamma\right) \times \operatorname{Br}\left(h_{2} \rightarrow h h\right) \times B r(h \rightarrow b \bar{b})^{2}$. For $m_{h}=125 \mathrm{GeV}$, the Higgs boson branching ratio decaying into $b \bar{b}$ is 0.584 [65]. We note that the initial state radiation (ISR) affects the signal and background cross sections. In calculating the cross sections, the Jadach-Ward-Was formalism [66] is used to consider the ISR effects.

The main background processes to this final state are:

$$
\begin{aligned}
& e^{+} e^{-} \rightarrow 4 b+\gamma, \\
& e^{+} e^{-} \rightarrow 2 b+2 j+\gamma(j=u, d, s, c, g), \text { two jets }(j) \text { are misidentified as b-jets, } \\
& e^{+} e^{-} \rightarrow 4 j+\gamma(j=u, d, s, c, g), \text { four jets }(j) \text { are misidentified as b-jets, }
\end{aligned}
$$

where all processes with off-shell $\gamma, Z, W^{ \pm}$and gluons are included.

The background rates are computed using COMPHEP [67] and MADGraPh5-AMC@NLO packages [68]. The efficiency of b-tagging for a jet originating from the hadronization of a bottom quark is taken $70 \%$, and misidentification rates of $10 \%$ and $1.5 \%$ are assumed for charm quark and light-flavor jets [69]. To select signal and background events and achieve a good sensitivity, the following requirements are applied: energy $(E)$ and pseudorapidity $\left(\eta=-\ln \tan \left(\frac{\theta}{2}\right)\right)$ of b-jets are required to be larger than $20 \mathrm{GeV}$ and $\left|\eta_{\mathrm{b}-\text { jet }}\right|<2.5$, respectively. Since the photon is expected to carry a large amount of energy due to its recoil against the heavy scalar $h_{2}$, its energy is required to be greater than $300 \mathrm{GeV}$ and $\left|\eta_{\gamma}\right|<2.5$. The signal and background efficiencies after these cuts are found to be $20.02 \%$ and $0.67 \%$, respectively. As in the signal events, the $b \bar{b}$ pairs come from the Higgs boson decays, it is required $100 \mathrm{GeV} \leq m_{b \bar{b}} \leq 150 \mathrm{GeV}$. This requirement has a strong power to suppress the background processes where the final state jets are not originating from Higgs bosons decays. For instance, it provides a rejection rate at the order of $\lesssim 10^{-5}$ for the major background processes, i.e. $e^{-}+e^{+} \rightarrow 4 b+\gamma$.

It should be mentioned that there are background processes in which an additional jet is misidentified as a photon. Such a signature may occur when neutral pions with large boost appear from jet fragmentation and decay to two photons. The showers from two photons can overlap in the electromagnetic calorimeter (ECAL) and will be observed as a single photon. The probability for a jet to be misidentified as a photon is dependent on the photon energy and is of the order of $10^{-5,-4}[70]$ for an energetic fake photon. Therefore, requiring a photon with $E \geq 300 \mathrm{GeV}$ suppresses the fake background contribution to a negligible level. A realistic detector simulation is necessary to estimate the fake background contribution.

In Fig,7, the 95\% CL excluded regions for $v_{H} / v_{S}$ versus $s_{\theta}$ and $v_{H} / v_{S}$ versus $m_{t} / m_{T}$ for the center-of-mass energy of $3 \mathrm{TeV}$ with an integrated luminosity of $3 \mathrm{ab}^{-1}$ are presented. The excluded regions are depicted for two scenarios of background contributions: scenario I where no uncertainty is considered on the number of expected background and scenario II where an uncertainty of $50 \%$ is taken on the number of expected backgrounds. The dot-dashed red curves in Figs 7 show the contours of favoured region at 95\% CL extracted from the precision electroweak data and Higgs boson measurements at the LHC [18]. As it can be seen, with the proposed selection in this work, for any value of the sine of mixing angle $s_{\theta} \in[0.05,0.3]$, any value of $v_{H} / v_{S}$ above 0.55 , corresponding to $v_{S} \lesssim 450 \mathrm{GeV}$, can be excluded. This would be a considerable improvement with respect to current bounds from electroweak precision tests and LHC Higgs data. The 95\% CL excluded region for $v_{H} / v_{S}$ versus $m_{t} / m_{T}$ indicates that a part of allowed region from electroweak precision tests and Higgs data corresponding to large mass of VLQ is accessible via the $h_{2} \gamma$ channel. For example, any value of $v_{S} \lesssim 1 \mathrm{TeV}$ can be excluded for $m_{T}=1.4 \mathrm{TeV}$.

In Fig. 8, the excluded regions of the parameter space in the plane of $\left(s_{\theta}^{2}, m_{h_{2}}\right)$ at $95 \%$ CL are shown. The results are presented for two scenarios of the expected background and are compared with those derived from $36 \mathrm{fb}^{-1}$ of LHC data at $13 \mathrm{TeV}, e^{-} e^{+} \rightarrow h_{2}+\nu \bar{\nu}$ process [56 at CLIC, 

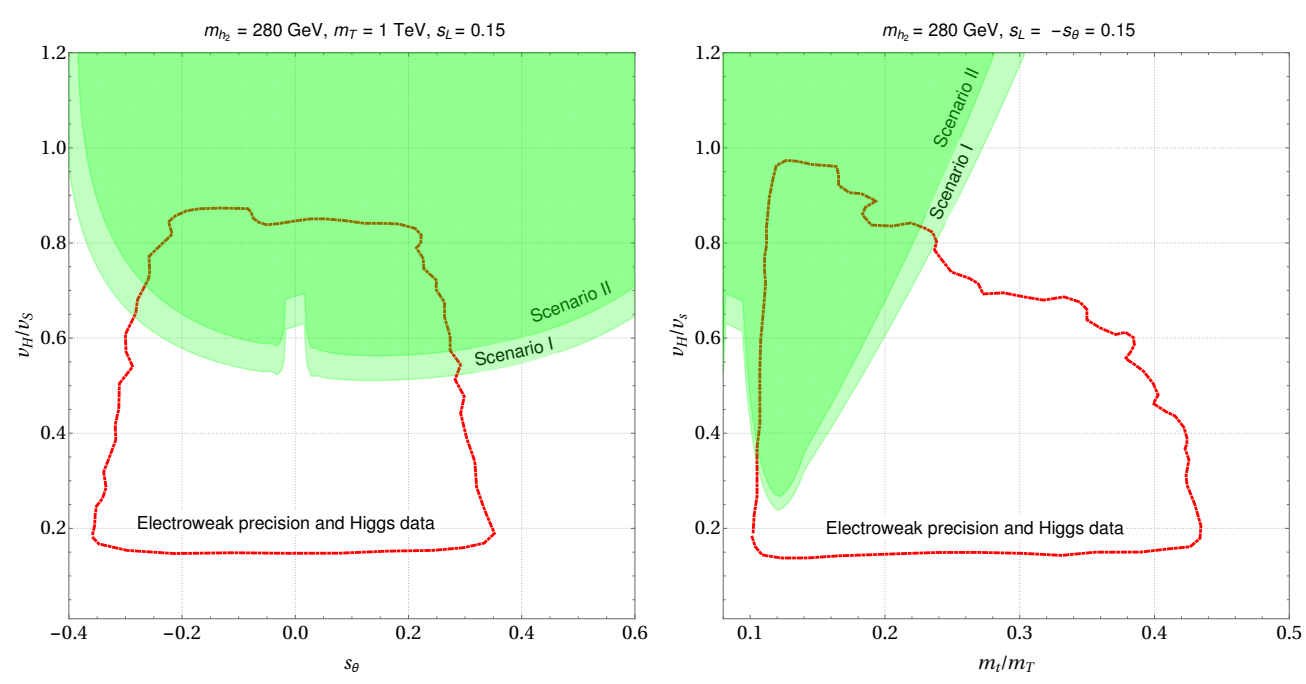

Figure 7: The green areas are the 95\% CL excluded regions of the parameter space in the planes of $\left(v_{H} / v_{S}, s_{\theta}\right)$ (left) and $\left(v_{H} / v_{S}, m_{t} / m_{T}\right)$ (right) for $\sqrt{s}=3 \mathrm{TeV}$ with $\mathcal{L}=3 \mathrm{ab}^{-1}$. The results are presented assuming that $m_{T}=1 \mathrm{TeV}$ and $m_{h_{2}}=280 \mathrm{GeV}$. The dot-dashed red curves represent contours of favoured region at 95\% CL obtained from the precision electroweak and Higgs data from LHC [18].

and the high luminosity LHC (HL-LHC) [56]. As it can be seen, using the $e^{-} e^{+} \rightarrow h_{2}+\nu \bar{\nu}$ process at the center-of-mass energy of $3 \mathrm{TeV}$ with an integrated luminosity of $3 \mathrm{ab}^{-1}$, any value of $s_{\theta}^{2}$ above $\sim 0.0015$ for $m_{h_{2}} \sim 300 \mathrm{GeV}$ could be excluded. The HL-LHC would be able to exclude $s_{\theta}^{2} \gtrsim 0.005$ for $m_{h_{2}} \sim 300 \mathrm{GeV}$ using the scalar decay into di-boson [56]. The associated production of $h_{2}$ with a photon is sensitive to a mass region of $m_{h_{2}} \sim 1200 \mathrm{GeV}$ for the values of $s_{\theta}^{2} \lesssim 0.15$. Considering no uncertainty would provide access to a mass region of $m_{h_{2}} \lesssim 1200$ $\mathrm{GeV}$ and taking into account an uncertainty of $50 \%$ on the expected background would reduce the sensitivity to mass, with almost $800 \mathrm{GeV}$ for $s_{\theta}^{2} \lesssim 0.1$. As we can see, the $h_{2} \gamma$ process would be able to scan a remarkable region of $s_{\theta}^{2} \lesssim 0.02$ with $m_{h_{2}} \lesssim 800 \mathrm{GeV}$ where the HL-LHC is not sensitive to. From Fig. 8, we also see that the excluded region derived from $h_{2} \gamma$ plays a complementary role to single scalar production at CLIC [56]. In particular, the $h_{2} \gamma$ process could probe the model at a mass region of $m_{h_{2}} \lesssim 1100 \mathrm{GeV}$, with $s_{\theta}^{2} \lesssim 0.001$ which is not accessible by the single scalar production.

The presented results here are at the center-of-mass energy of $3 \mathrm{TeV}$ and are based on an integrated luminosity of $3 \mathrm{ab}^{-1}$ which is expected to be attainable by CLIC according to Ref. 71. However, it should be noted that different center-of-mass energies are sensitive to a particular part of the model parameters. For example, as the left plot of Fig 5 shows, the total cross section of $h_{2} \gamma$ process is maximum at $\sqrt{s}=1 \mathrm{TeV}$ for $m_{T}=v_{S}=500 \mathrm{GeV}$ while with $m_{T}=1 \mathrm{TeV}, v_{S}=350$ $\mathrm{GeV}$, collisions at $\sqrt{s}=3 \mathrm{TeV}$ provides the largest cross section. Therefore, performing the study at different center-of-mass energies and combination would allow to extend the sensitivity to larger part of the parameter space.

In this investigation, we only concentrated on di-Higgs decay mode of $h_{2}$, followed by $h \rightarrow b \bar{b}$, and cuts on few kinematic variables are considered to reduce the backgrounds. However, there are rooms for improving the sensitivity which could be achieved by (i) considering other decay modes of the Higgs boson as mentioned in the last column of Table 1. (ii) using the other decay channels of $h_{2}$ like $h_{2} \rightarrow W W, Z Z, t \bar{t}$ and combination of all channels together; (iii) using various kinematic variables to suppress the contributions and also exploiting sophisticated methods such 

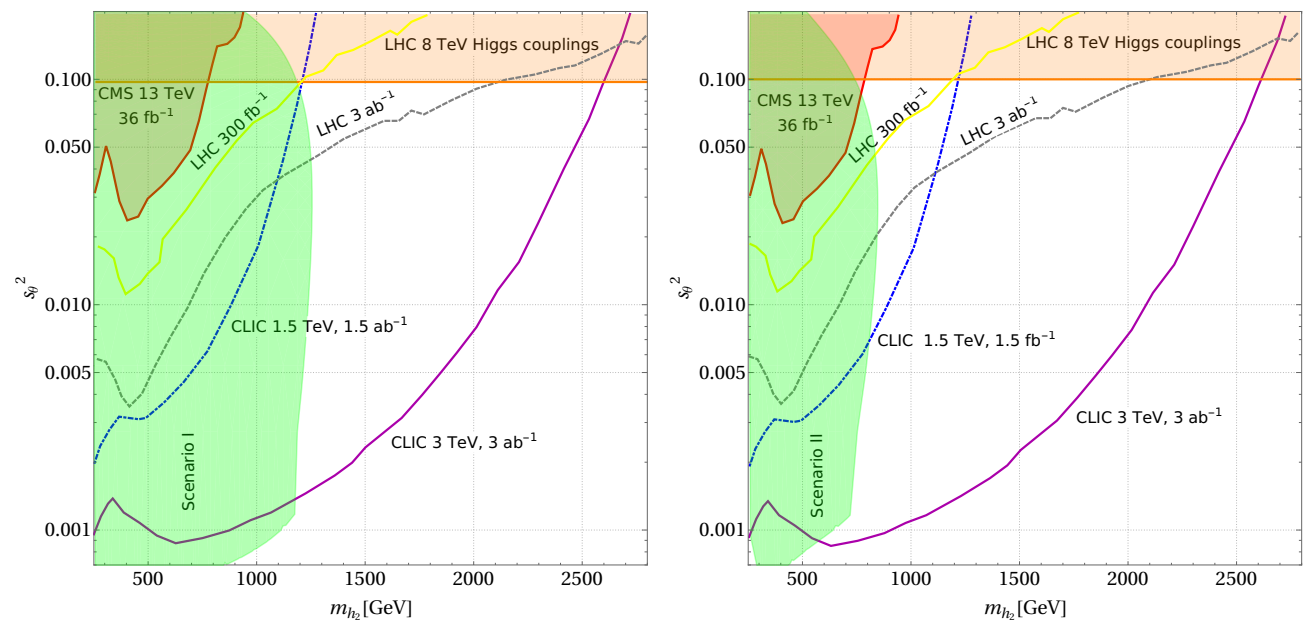

Figure 8: The green areas are the 95\% CL excluded regions of the parameter space in the plane of $\left(s_{\theta}^{2}, m_{h_{2}}\right)$ with no (left) and with $50 \%$ (right) uncertainty on the expected number of background events. These regions are derived from $e^{-} e^{+} \rightarrow h_{2} \gamma$ with subsequent $h_{2}$ decay into di-Higgs and Higgs decays into $b \bar{b}$. The results are shown under assumption of $m_{T}=1 \mathrm{TeV}, s_{L}=0.15$ and $v_{S}=350 \mathrm{GeV}$. The red and orange shaded regions obtained from LHC direct search with the scalar decays into di-boson and from indirect searches. The constraints from CLIC at $\sqrt{s}=1.5 \mathrm{TeV}$ and $1.5 \mathrm{ab}^{-1}$ (dot-dashed blue) and $\sqrt{s}=3 \mathrm{TeV}$ and $3 \mathrm{ab}^{-1}$, obtained from the single scalar channel, are depicted with blue and purple curves. The constraints from the HL-LHC with a luminosity of $300 \mathrm{fb}^{-1}$ (yellow) and $3 \mathrm{ab}^{-1}$ (dashed gray) are presented for comparison as well 56 .

as multivariate techniques to distinguish signal events from background events.

\section{Summary}

To summarise, we performed an analytic calculation for the production cross section of a neutral singlet scalar heavier than the observed SM Higgs boson in association with a photon $\left(h_{2} \gamma\right)$ in the context of a simplified SM extension model, at a lepton collider. In this model the scalar mixes with the SM Higgs boson and can couple to a pair of SM massless bosons, i.e. photon and gluon, at loop level via a vector like fermion which mixes with the top quark. Such a model provides the possibility to stabilise the electroweak vacuum [9].

We presented the size of the $h_{2} \gamma$ cross section for various center-of-mass energies which would be reached at future lepton colliders. The cross section is calculated in terms of the model parameters and in general it was found to be small but the signal signatures are rather clean and could be used to span the parameter space of the model. To examine the potential of the process to explore the model, we concentrated on the decay of $h_{2}$ to $h h$ followed by the Higgs bosons decays into $b \bar{b}$ pairs. Using a set of selection cuts for the signal events which efficiently suppress the background contributions, the exclusion regions at 95\% CL are derived. This was done as an example at the center-of-mass energy of $\sqrt{s}=3 \mathrm{TeV}$ with an integrated luminosity of $3 \mathrm{ab}^{-1}$. Overall, the results indicate that the associated production of the scalar $h_{2}$ with a photon is sensitive to a part of the parameter space with $m_{h_{2}} \lesssim 1.2 \mathrm{TeV}$ and low values of the mixing angle which is out of access at the HL-LHC. A comparison of the results with the single scalar production at CLIC suggests that the $h_{2} \gamma$ process has the ability to complement the single scalar channel at very low values of the mixing angle. 
Acknowledgments: M. Mohammadi Najafabadi is grateful to INSF for the support. S. Tizchang is grateful to Hiren Patel and Rodolfo Capdevilla for valuable help in calculations with packages in Mathematica, and F. Elahi for fruitful discussions.

\section{A Appendix}

The $F$ functions appeared in Eq20 are defined as:

$$
\begin{aligned}
F^{a} & =4\left[\mathbf{C}_{22}^{a}+\mathbf{C}_{12}^{a}+\mathbf{C}_{2}^{a}+\frac{\mathbf{C}_{0}^{a}}{4}\right], \\
F^{n} & =2\left(m_{t}+m_{T}\right)\left(\mathbf{C}_{22}^{n}+\mathbf{C}_{12}^{n}\right)+m_{T} \mathbf{C}_{0}^{n}+\left(m_{t}+3 m_{T}\right) \mathbf{C}_{2}^{n}-\left(m_{t}-m_{T}\right) \mathbf{C}_{1}^{n}, \\
F^{l} & =F^{n}\left[m_{t} \leftrightarrow m_{T}, n \rightarrow l\right],
\end{aligned}
$$

where $a=f, t, T$ and $\mathbf{C}_{i j}$ s are the common scalar two and three point Passarino-Veltman functions which are defined as:

$$
\begin{aligned}
\mathbf{C}_{i j}^{f} & \equiv \mathbf{C}_{i j}\left(s, 0, m_{h_{2}}^{2}, m_{f}^{2}, m_{f}^{2}, m_{f}^{2}\right), \\
\mathbf{C}_{i j}^{t} & \equiv \mathbf{C}_{i j}\left(s, 0, m_{h_{2}}^{2}, m_{t}^{2}, m_{t}^{2}, m_{t}^{2}\right), \\
\mathbf{C}_{i j}^{T} & \equiv \mathbf{C}_{i j}\left(s, 0, m_{h_{2}}^{2}, m_{T}^{2}, m_{T}^{2}, m_{T}^{2}\right), \\
\mathbf{C}_{i j}^{n} & \equiv \mathbf{C}_{i j}\left(s, 0, m_{h_{2}}^{2}, m_{t}^{2}, m_{T}^{2}, m_{T}^{2}\right), \\
\mathbf{C}_{i j}^{l} & \equiv \mathbf{C}_{i j}\left(s, 0, m_{h_{2}}^{2}, m_{T}^{2}, m_{t}^{2}, m_{t}^{2}\right) .
\end{aligned}
$$

In Eq $20, F^{\gamma / Z, W}$ are the combination of Passarino-Veltman scalar functions:

$$
\begin{aligned}
& F^{\gamma, W}=4\left(\frac{m_{h_{2}}^{2}}{M_{W}^{2}}+6\right)\left(\mathbf{C}_{12}+\mathbf{C}_{22}+\mathbf{C}_{2}\right)+16 \mathbf{C}_{0}, \\
& F^{Z, W}=2\left[\frac{m_{h_{2}}^{2}}{M_{W}^{2}}\left(1-2 c_{w}^{2}\right)+2\left(1-6 c_{w}^{2}\right)\right]\left(\mathbf{C}_{22}+\mathbf{C}_{12}+\mathbf{C}_{2}\right)+4\left(1-4 c_{w}^{2}\right) \mathbf{C}_{0},
\end{aligned}
$$

with

$$
\mathbf{C}_{i j} \equiv \mathbf{C}_{i j}\left(s, 0, m_{h_{2}}^{2}, M_{W}^{2}, M_{W}^{2} M_{W}^{2}\right) .
$$

The $C_{1,2}^{ \pm \text {Wbox }}$ and $C_{1,2}^{ \pm \text {Zbox }}$ in Eq, 17 have the following forms:

$$
\begin{aligned}
C_{1}^{+ \text {Wbox }} & =-\frac{e^{4} M_{W} s_{\theta}}{s_{w}^{3}}\left[\mathbf{D}_{1}^{a}+\mathbf{D}_{1}^{b}+\mathbf{D}_{13}^{b}-\mathbf{D}_{13}^{a}-\mathbf{D}_{33}^{a}\right] \\
C_{2}^{+ \text {Wbox }} & =-\frac{e^{4} M_{W} s_{\theta}}{s_{w}^{3}}\left[\mathbf{D}_{2}^{a}+\mathbf{D}_{23}^{a}+\mathbf{D}_{2}^{b}-\mathbf{D}_{23}^{b}-\mathbf{D}_{33}^{b}\right] \\
C_{1,2}^{- \text {Wbox }} & =0, \\
C_{1}^{ \pm \text {Zbox }} & =-\frac{2 e^{4} M_{W} s_{\theta} g_{e}^{\mp 2}}{s_{w} c_{w}^{2}}\left[\mathbf{D}_{13}^{c}+\mathbf{D}_{33}^{c}\right] \\
C_{2}^{ \pm \text {Zbox }} & =\frac{2 e^{4} M_{W} s_{\theta} g_{e}^{\mp 2}}{s_{w} c_{w}^{2}}\left[\mathbf{D}_{2}^{c}+\mathbf{D}_{12}^{c}+\mathbf{D}_{23}^{c}\right]
\end{aligned}
$$


where $g_{e}^{-} \equiv I_{e}^{3} /\left(s_{w} c_{w}\right)-Q_{e} s_{w} / c_{w}$, and $g_{e}^{+} \equiv-Q_{e} s_{w} / c_{w}$. Four point Passarino-Veltman functions $\mathbf{D}_{i j}$ are defined as follows:

$$
\begin{aligned}
& \mathbf{D}_{i j}^{a} \equiv \mathbf{D}_{i j}\left(0, s, m_{h_{2}}^{2}, u, 0,0,0, M_{W}^{2}, M_{W}^{2}, M_{W}^{2}\right) \\
& \mathbf{D}_{i j}^{b} \equiv \mathbf{D}_{i j}\left(0, s, 0, t, 0, m_{h_{2}}^{2}, 0, M_{W}^{2}, M_{W}^{2}, M_{W}^{2}\right) \\
& \mathbf{D}_{i j}^{c} \equiv \mathbf{D}_{i j}\left(0, u, m_{h_{2}}^{2}, t, 0,0,0,0, M_{Z}^{2}, M_{Z}^{2}\right)
\end{aligned}
$$

\section{References}

[1] G. Aad et al. [ATLAS Collaboration], Phys. Lett. $\quad$ B $\mathbf{7 1 6 ,} 1$ (2012) doi:10.1016/j.physletb.2012.08.020 arXiv:1207.7214 [hep-ex]].

[2] S. Chatrchyan et al. [CMS Collaboration], Phys. Lett. B 716, 30 (2012) doi:10.1016/j.physletb.2012.08.021 arXiv:1207.7235 [hep-ex]].

[3] V. Khachatryan et al. [CMS Collaboration], Eur. Phys. J. C 75, no. 5, 212 (2015) doi:10.1140/epjc/s10052-015-3351-7 arXiv:1412.8662 [hep-ex]].

[4] G. Aad et al. [ATLAS Collaboration], Eur. Phys. J. C 75, no. 10, 476 (2015) Erratum: [Eur. Phys. J. C 76, no. 3, 152 (2016)] doi:10.1140/epjc/s10052-015-3685-1, 10.1140/epjc/s10052016-3934-y arXiv:1506.05669 [hep-ex]].

[5] G. Aad et al. [ATLAS Collaboration], Eur. Phys. J. C 76, no. 1, 6 (2016) doi:10.1140/epjc/s10052-015-3769-y arXiv:1507.04548 [hep-ex]].

[6] V. Khachatryan et al. [CMS Collaboration], Phys. Rev. D 92, no. 7, 072010 (2015) doi:10.1103/PhysRevD.92.072010 [arXiv:1507.06656 [hep-ex]].

[7] J. Cao, Y. He, P. Wu, M. Zhang and J. Zhu, JHEP 1401, 150 (2014) doi:10.1007/JHEP01(2014)150 arXiv:1311.6661 [hep-ph]].

[8] B. Batell, S. Jung and H. M. Lee, JHEP 1301, 135 (2013) doi:10.1007/JHEP01(2013)135 arXiv:1211.2449 [hep-ph]].

[9] M. L. Xiao and J. H. Yu, Phys. Rev. D 90, no. 1, 014007 (2014) Addendum: [Phys. Rev. D 90, no. 1, 019901 (2014)] doi:10.1103/PhysRevD.90.014007, 10.1103/PhysRevD.90.019901 arXiv:1404.0681 [hep-ph]].

[10] S. Fajfer, A. Greljo, J. F. Kamenik and I. Mustac, JHEP 1307, 155 (2013) doi:10.1007/JHEP07(2013)155 arXiv:1304.4219 [hep-ph]].

[11] G. Abbas, arXiv:1712.08052 [hep-ph].

[12] D. B. Kaplan and H. Georgi, Phys. Lett. 136B, 183 (1984). doi:10.1016/0370-2693(84)911778 ; D. B. Kaplan, H. Georgi and S. Dimopoulos, Phys. Lett. 136B, 187 (1984). doi:10.1016/0370-2693(84)91178-X 
[13] N. Gutierrez Ortiz, J. Ferrando, D. Kar and M. Spannowsky, Phys. Rev. D 90, no. 7, 075009 (2014) doi:10.1103/PhysRevD.90.075009 [arXiv:1403.7490 [hep-ph]].

[14] I. Antoniadis, Phys. Lett. B 246, 377 (1990). doi:10.1016/0370-2693(90)90617-F

[15] N. Arkani-Hamed, A. G. Cohen, E. Katz, A. E. Nelson, T. Gregoire and J. G. Wacker, JHEP 0208, 021 (2002) doi:10.1088/1126-6708/2002/08/021 [hep-ph/0206020].

[16] Y. Grossman and M. Neubert, Phys. Lett. B 474, 361 (2000) doi:10.1016/S03702693(00)00054-X hep-ph/9912408].

[17] T. Moroi and Y. Okada, Mod. Phys. Lett. A 7, 187 (1992). doi:10.1142/S0217732392000124

[18] M. J. Dolan, J. L. Hewett, M. Kramer and T. G. Rizzo, JHEP 1607, 039 (2016) doi:10.1007/JHEP07(2016)039 [arXiv:1601.07208 [hep-ph]].

[19] D. Buttazzo, G. Degrassi, P. P. Giardino, G. F. Giudice, F. Sala, A. Salvio and A. Strumia, JHEP 1312, 089 (2013) doi:10.1007/JHEP12(2013)089 [arXiv:1307.3536 [hep-ph]].

[20] G. Isidori, G. Ridolfi and A. Strumia, Nucl. Phys. B 609, 387 (2001) doi:10.1016/S05503213(01)00302-9 hep-ph/0104016.

[21] L. A. Anchordoqui, I. Antoniadis, H. Goldberg, X. Huang, D. Lust, T. R. Taylor and B. Vlcek, JHEP 1302, 074 (2013) doi:10.1007/JHEP02(2013)074 [arXiv:1208.2821 [hep-ph]].

[22] G. Degrassi, S. Di Vita, J. Elias-Miro, J. R. Espinosa, G. F. Giudice, G. Isidori and A. Strumia, JHEP 1208, 098 (2012) doi:10.1007/JHEP08(2012)098 arXiv:1205.6497 [hep-ph]].

[23] J. Elias-Miro, J. R. Espinosa, G. F. Giudice, G. Isidori, A. Riotto and A. Strumia, Phys. Lett. B 709, 222 (2012) doi:10.1016/j.physletb.2012.02.013 arXiv:1112.3022 [hep-ph]].

[24] G. Isidori, Y. Nir and G. Perez, Ann. Rev. Nucl. Part. Sci. 60, 355 (2010) doi:10.1146/annurev.nucl.012809.104534 [arXiv:1002.0900 [hep-ph]].

[25] H. Han, S. Wang and S. Zheng, Nucl. Phys. B 907, $180 \quad$ (2016) doi:10.1016/j.nuclphysb.2016.04.002 arXiv:1512.06562 [hep-ph]].

[26] A. Falkowski, O. Slone and T. Volansky, JHEP 1602, $152 \quad$ (2016) doi:10.1007/JHEP02(2016)152 arXiv:1512.05777 [hep-ph]].

[27] S. Knapen, T. Melia, M. Papucci and K. Zurek, Phys. Rev. D 93, no. 7, 075020 (2016) doi:10.1103/PhysRevD.93.075020 [arXiv:1512.04928 [hep-ph]].

[28] J. Ellis, S. A. R. Ellis, J. Quevillon, V. Sanz and T. You, JHEP 1603, 176 (2016) doi:10.1007/JHEP03(2016)176 arXiv:1512.05327 [hep-ph]].

[29] R. Benbrik, C. H. Chen and T. Nomura, Phys. Rev. D 93, no. 5, 055034 (2016) doi:10.1103/PhysRevD.93.055034 arXiv:1512.06028 [hep-ph]].

[30] J. Zhang and S. Zhou, Chin. Phys. C 40, no. 8, 081001 (2016) doi:10.1088/16741137/40/8/081001 arXiv:1512.07889 [hep-ph]].

[31] R. S. Gupta, S. Jager, Y. Kats, G. Perez and E. Stamou, JHEP 1607, 145 (2016) doi:10.1007/JHEP07(2016)145 arXiv:1512.05332 [hep-ph]]. 
[32] N. Craig, P. Draper, C. Kilic and S. Thomas, Phys. Rev. D 93, no. 11, 115023 (2016) doi:10.1103/PhysRevD.93.115023 arXiv:1512.07733 [hep-ph]].

[33] V. Khachatryan et al. [CMS Collaboration], Phys. Rev. D 93, no. 1, 012003 (2016) doi:10.1103/PhysRevD.93.012003 [arXiv:1509.04177 [hep-ex]].

[34] G. Aad et al. [ATLAS Collaboration], JHEP 1508, 105 (2015) doi:10.1007/JHEP08(2015)105 arXiv:1505.04306 [hep-ex]].

[35] J. P. Araque [ATLAS Collaboration], arXiv:1611.09056 [hep-ex].

[36] D. Barducci et al., JHEP 1412, 080 (2014) doi:10.1007/JHEP12(2014)080 arXiv:1405.0737 [hep-ph]].

[37] S. Banerjee, D. Barducci, G. Belanger and C. Delaunay, JHEP 1611, 154 (2016) doi:10.1007/JHEP11(2016)154 arXiv:1606.09013 [hep-ph]].

[38] M. Buchkremer, G. Cacciapaglia, A. Deandrea and L. Panizzi, Nucl. Phys. B 876, 376 (2013) doi:10.1016/j.nuclphysb.2013.08.010 arXiv:1305.4172 [hep-ph]].

[39] G. Cacciapaglia, A. Deandrea, N. Gaur, D. Harada, Y. Okada and L. Panizzi, JHEP 1509, 012 (2015) doi:10.1007/JHEP09(2015)012 [arXiv:1502.00370 [hep-ph]].

[40] G. Cacciapaglia, A. Deandrea, L. Panizzi, S. Perries and V. Sordini, JHEP 1303, 004 (2013) doi:10.1007/JHEP03(2013)004 [arXiv:1211.4034 [hep-ph]].

[41] J. A. Aguilar-Saavedra, R. Benbrik, S. Heinemeyer and M. Perez-Victoria, Phys. Rev. D 88, no. 9, 094010 (2013) doi:10.1103/PhysRevD.88.094010 arXiv:1306.0572 [hep-ph]].

[42] J. A. Aguilar-Saavedra, JHEP 0911, 030 (2009) doi:10.1088/1126-6708/2009/11/030 arXiv:0907.3155 [hep-ph]].

[43] H. Alhazmi, J. H. Kim, K. Kong and I. M. Lewis, arXiv:1808.03649 [hep-ph]. ; J. H. Kim and I. M. Lewis, JHEP 1805, 095 (2018) doi:10.1007/JHEP05(2018)095 arXiv:1803.06351 [hep-ph]].

[44] A. M. Sirunyan et al. [CMS Collaboration], Phys. Lett. B 781, 574 (2018) doi:10.1016/j.physletb.2018.04.036 [arXiv:1708.01062 [hep-ex]].

[45] M. Aaboud et al. [ATLAS Collaboration], arXiv:1806.10555 [hep-ex].

[46] K. Fujii et al., arXiv:1506.05992 [hep-ex].

[47] T. Barklow, J. Brau, K. Fujii, J. Gao, J. List, N. Walker and K. Yokoya, arXiv:1506.07830 [hep-ex].

[48] M. J. Boland et al. [CLIC and CLICdp Collaborations], doi:10.5170/CERN-2016-004 arXiv:1608.07537 [physics.acc-ph].

[49] M. Bicer et al. [TLEP Design Study Working Group], JHEP 1401, 164 (2014) doi:10.1007/JHEP01(2014)164 [arXiv:1308.6176 [hep-ex]].

[50] M. Koratzinos, A. Blondel, E. Gianfelice-Wendt and F. Zimmermann, arXiv:1506.00933 [physics.acc-ph]. 
[51] CEPC-SPPC Study Group, IHEP-CEPC-DR-2015-01, IHEP-TH-2015-01, IHEP-EP-201501.

[52] A. Ilnicka, T. Robens and T. Stefaniak, Mod. Phys. Lett. A 33, no. 10n11, 1830007 (2018) doi:10.1142/S0217732318300070 [arXiv:1803.03594 [hep-ph]].

[53] G. Dupuis, JHEP 1607, 008 (2016) doi:10.1007/JHEP07(2016)008 arXiv:1604.04552 [hep$\mathrm{ph}]$.

[54] S. Dawson and E. Furlan, Phys. Rev. D 86 (2012) 0150211205.4733.

[55] J. A. Aguilar-Saavedra, R. Benbrik, S. Heinemeyer and M. Perez-Victoria, Phys. Rev. D 88, no. 9, 094010 (2013) doi:10.1103/PhysRevD.88.094010 [arXiv:1306.0572 [hep-ph]].

[56] D. Buttazzo, D. Redigolo, F. Sala and A. Tesi, JHEP 1811, 144 (2018) doi:10.1007/JHEP11(2018)144 arXiv:1807.04743 [hep-ph]].

[57] W. F. Chang, T. Modak and J. N. Ng, Phys. Rev. D 97, no. 5, 055020 (2018) doi:10.1103/PhysRevD.97.055020 [arXiv:1711.05722 [hep-ph]].

[58] A. Djouadi, V. Driesen, W. Hollik and J. Rosiek, Nucl. Phys. B 491, 68 (1997) doi:10.1016/S0550-3213(96)00711-0 [hep-ph/9609420].

[59] S. Kanemura, K. Mawatari and K. Sakurai, arXiv:1808.10268 [hep-ph].

[60] H. Khanpour, S. Khatibi and M. Mohammadi Najafabadi, Phys. Lett. B 773, 462 (2017) doi:10.1016/j.physletb.2017.09.005 [arXiv:1702.05753 [hep-ph]].

[61] W. L. Sang, W. Chen, F. Feng, Y. Jia and Q. F. Sun, Phys. Lett. B 775, 152 (2017) doi:10.1016/j.physletb.2017.10.044 [arXiv:1706.03572 [hep-ph]].

[62] H. H. Patel, Comput. Phys. Commun. 197, 276 (2015) doi:10.1016/j.cpc.2015.08.017 arXiv:1503.01469 [hep-ph]].

[63] T. Hahn and M. Perez-Victoria, Comput. Phys. Commun. 118, 153 (1999) doi:10.1016/S00104655(98)00173-8 hep-ph/9807565].

[64] K. Nakamura, K. Nishiwaki, K. y. Oda, S. C. Park and Y. Yamamoto, Eur. Phys. J. C 77, no. 5, 273 (2017) doi:10.1140/epjc/s10052-017-4835-4 arXiv:1701.06137 [hep-ph]].

[65] C. Patrignani et al. [Particle Data Group], Chin. Phys. C 40, no. 10, 100001 (2016). doi:10.1088/1674-1137/40/10/100001

[66] S. Jadach, B. F. L. Ward and Z. Was, Phys. Rev. D 63, 113009 (2001) doi:10.1103/PhysRevD.63.113009 hep-ph/0006359].

[67] E. Boos et al. [CompHEP Collaboration], Nucl. Instrum. Meth. A 534, 250 (2004) doi:10.1016/j.nima.2004.07.096 hep-ph/0403113.

[68] J. Alwall et al., JHEP 1407, 079 (2014) doi:10.1007/JHEP07(2014)079 arXiv:1405.0301 [hep-ph]].

[69] D. Arominski et al. [CLICdp Collaboration], arXiv:1812.07337 [physics.ins-det]. 
[70] S. D. Ellis, T. S. Roy and J. Scholtz, Phys. Rev. D 87, no. 1, 014015 (2013) doi:10.1103/PhysRevD.87.014015 [arXiv:1210.3657 [hep-ph]].

[71] H. Abramowicz et al., Eur. Phys. J. C 77, no. 7, 475 (2017) doi:10.1140/epjc/s10052-0174968-5 arXiv:1608.07538 [hep-ex]]. 